\title{
Laminar flow in chevron-type plate heat exchangers: CFD analysis of tortuosity, shape factor and friction factor
}

\author{
Carla S. Fernandes ${ }^{a}, *$, Ricardo P. Dias ${ }^{b, 1}$, João M. Nóbrega ${ }^{\mathrm{c}, 2}$, João M. Maia ${ }^{\mathrm{c}, 2}$ \\ a Departamento de Matemática, Escola Superior de Tecnologia e de Gestão, Instituto Politécnico de Bragança, \\ Campus de Santa Apolónia, 5301-857 Bragança, Portugal \\ ${ }^{\mathrm{b}}$ Departamento de Tecnologia Química e Biológica, Escola Superior de Tecnologia e de Gestão, Instituto Politécnico de Bragança, \\ Campus de Santa Apolónia, 5301-857 Bragança, Portugal \\ ${ }^{\mathrm{c}}$ Institute for Polymers and Composites (IPC), Departamento de Engenharia de Polímeros, Universidade do Minho, $4800-058$ Guimarães, Portugal \\ Received 5 January 2007; received in revised form 9 April 2007; accepted 26 May 2007 \\ Available online 6 June 2007
}

\begin{abstract}
Laminar or low Reynolds number flows are usually obtained when liquid foods with high viscosity are processed in plate heat exchangers (PHEs). The tortuosity coefficient is a key parameter used by PHEs manufacturers to estimate Fanning friction factors and convective heat transfer coefficients. Using the finite-element computational fluid dynamics program POLYFLOW ${ }^{\circledR}$, fully developed laminar flows in double-sine chevrontype PHEs passages are analysed in this work. The corrugation angle and channel aspect ratio of the passages vary in a broad range, PHEs with common area enlargement factors and with high area density being studied. The tortuosity coefficient and the coefficient $K$ (Kozeny's coefficient in granular beds) from the friction factor correlations increase with the increase of the channels aspect ratio and the decrease of the chevron angle. The shape factor from the PHEs passages also increases with the decrease of the chevron angle and is weakly influenced by the channel aspect ratio. In this paper, relations to predict the tortuosity coefficient and shape factor are proposed, the coefficient $K$ being predicted resorting to the tortuosity coefficient and shape factor. The coefficient $K$ compares well with literature data in the region of common chevron angles, channels aspect ratio and area enlargement factor.
\end{abstract}

(c) 2007 Elsevier B.V. All rights reserved.

Keywords: Plate heat exchangers; Friction factor; Tortuosity; Shape factor

\section{Introduction}

\subsection{Laminar flow in plate heat exchangers}

Laminar flow in complex ducts geometries is of both fundamental and practical interest [1-6]. Of particular interest is the PHE which is extensively used in the chemical, pharmaceutical and food industries, among others [1,7,8]. Laminar or low Reynolds number flows are usually obtained when liquid foods are processed in PHEs, this low Reynolds number range being also observed in micro PHEs [7,9-12]. Besides the importance in pressure drops estimations, the development of Fanning fric-

\footnotetext{
* Corresponding author. Tel.: +351 273303 127; fax: +351 273313051 .

E-mail address: cveiga@ipb.pt (C.S. Fernandes).

1 Tel.: +351 273303 150; fax: +351273313051.

${ }^{2}$ Tel.: +351 253510 320; fax: +351253510339.
}

tion factor correlations, determined for the isothermal laminar flow of Newtonian fluids in PHEs, can be useful in other areas. One of them is the prediction of port-to-channel flow maldistribution in these equipments [13,14], the referred correlations being also involved in the development of methodologies that allow the establishment of a single friction curve equation for both Newtonian and power law fluids, in different flow regimes $[12,15]$.

In the referred methodologies, geometrical parameters of the ducts need to be estimated in order to define generalized Reynolds numbers. The geometrical parameters are estimated using Fanning friction factor expressions, determined for the laminar flow of Newtonian fluids in PHE passages [15] or other type of ducts [16].

Physical processing brings about irreversible textural and sensorial properties of nearly all the fluids in the food industry $[17,18]$. The above mentioned Fanning friction factor correlations can be helpful in the calculation of wall shear rates 
developed during the flow of Newtonian or power law fluids inside the PHE channels $[11,12,15]$. These wall shear rates can then be used to predict the viscosity breakdown of liquid foodstuffs during their processing [17].

Stirred yoghurt is very sensitive to physical processing, a low viscosity being a common manufacturing defect of this foodstuff [17]. During the flow through the cylindrical filling nozzles, the yoghurt is subjected to high wall shear rates (typical values are between 800 and $1250 \mathrm{~s}^{-1}$ ) and this may lead to an irreversible breakdown of yoghurt viscosity [17].

PHEs are commonly used during the cooling of stirred yoghurt [7,19]. In the work from Fernandes et al. [19], it can be observed that in a commercial PHE and for a Reynolds number of 12.3 wall shear rate reaches $1800 \mathrm{~s}^{-1}$, this value being substantially superior to the observed in the filling nozzles.

\subsection{Corrugation geometry}

Between the more than 60 different plate surface corrugation patterns, the most used PHEs consist of plates with chevrontype corrugations that have a sinusoidal shape [1] (Fig. 1). The thermal-hydraulic performance of PHEs is strongly dependent on the geometrical properties of the chevron plates [20-22], namely on the corrugation angle, $\beta$, area enlargement factor, $\phi$, defined as the ratio between the effective plate area and projected plate area, and channel aspect ratio (Fig. 1).

(a)

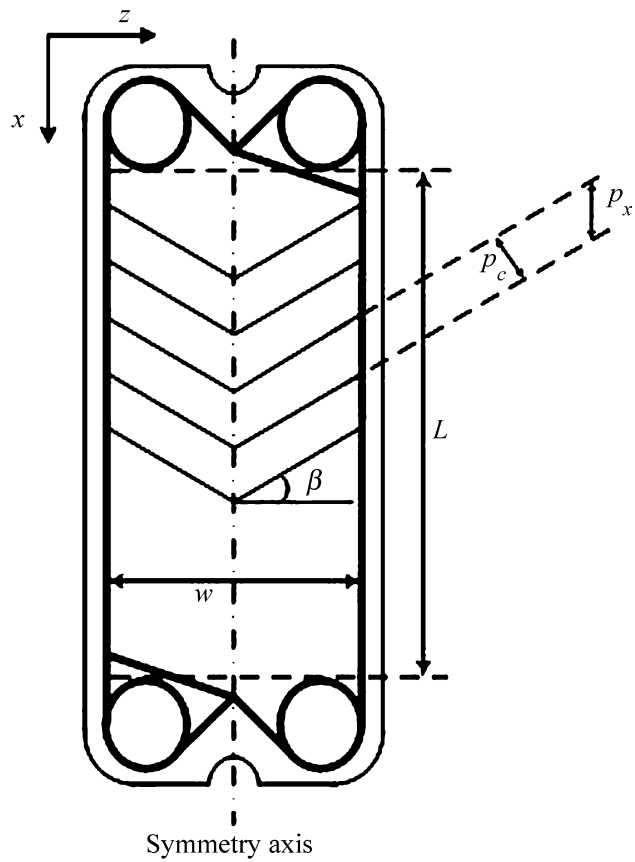

(b)

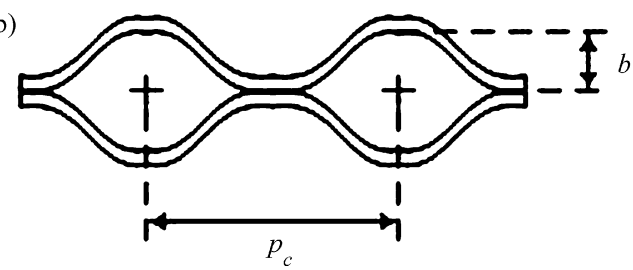

Fig. 1. (a) Schematic representation of a chevron plate; (b) corrugation dimensions.
The channel aspect ratio is usually defined by $2 b / p_{\mathrm{c}}, p_{\mathrm{c}}$ being the corrugation pitch and $b$ the inter-plates distance (Fig. 1). In the present work, a different definition of channel aspect ratio, $\gamma$, will be used:

$\gamma=\frac{2 b}{p_{x}}$

$p_{x}$ being the corrugation pitch in the main flow direction (Fig. 1). The reason for the use of a channel aspect ratio defined in the main flow direction is explained in Section 3.

Introducing Eq. (1) in the expression proposed by Martin [23] and resorting to the geometric relation between $p_{\mathrm{c}}$ and $p_{x}$ (Fig. 1), the area enlargement factor can be estimated by:

$$
\begin{aligned}
\phi= & \frac{1}{6}\left\{1+\left[1+\left(\frac{\pi}{2 \cos (\beta)}\right)^{2} \gamma^{2}\right]^{0.5}\right. \\
& \left.+4\left[1+\left(\frac{\pi}{2 \sqrt{2} \cos (\beta)}\right)^{2} \gamma^{2}\right]^{0.5}\right\}
\end{aligned}
$$

The area enlargement factor typically assumes values between 1.1 and 1.5 [22], $b$ normally lies in the range $2-5 \mathrm{~mm}[21,24]$ and $\beta$ is typically located in the range $22-65^{\circ}[21,22]$.

\subsection{Friction factor, shape factor and tortuosity coefficient}

Fanning friction factors correlations, $f R e$, in the laminar regime take the form $[21,22]$ :

$f=K \operatorname{Re}^{-1}$,

where $K$ is a coefficient dependent of the corrugation angle and channel aspect ratio and $R e$ the Reynolds number:

$R e=\frac{\rho u D_{\mathrm{H}}}{\eta}$

$\rho$ and $\eta$ representing the fluid density and viscosity, respectively. In Eq. (4) the mean velocity, $u$, in the PHE channel and the hydraulic diameter, $D_{\mathrm{H}}$, can be calculated by [21,22]:

$$
u=\frac{M_{\mathrm{v}}}{w b},
$$

and

$D_{\mathrm{H}}=\frac{4 \times \text { channel flow area }}{\text { wetted surface }} \cong \frac{2 b}{\phi}$,

where $M_{\mathrm{v}}$ is the volumetric flow rate and $w$ the channel width.

The Fanning friction factor, $f$, can be estimated by:

$f=\frac{\Delta P D_{\mathrm{H}}}{2 L \rho u^{2}}$,

where $\Delta P$ is the pressure drop and $L$ the length of the channel.

In the experimental studies of Kumar [20] it was found that the critical Reynolds number (the Reynolds number where laminar flow ends) increased with the increase of the corrugation angle. Using plates with chevron angles of $30^{\circ}$ and $65^{\circ}$ the author 
observed laminar flow for Reynolds numbers below 10 and 50, respectively.

The cross-corrugated passages from PHEs containing plates of the chevron-type are characterized by Kumar [20] as being tortuous. As the corrugation angle is reduced from $90^{\circ}-\mathrm{a}$ double-sine duct [1] - the passage becomes more tortuous and offers greater hydrodynamic resistance [20]. The pressure drop across a tortuous channel with a shape factor $K_{0}$ can be related with the average interstitial velocity, $v$, according to Hagen-Poiseuille's formula [25]:

$\Delta P=\frac{2 K_{0} \eta L_{\mathrm{av}} v}{D_{\mathrm{H}}^{2}}$,

where $L_{\mathrm{av}}$ is the average travel distance of a fluid element in the channel. The mean velocity and $v$ are related by [25]:

$v=u \frac{L_{\mathrm{av}}}{L}=u \tau$

$\tau$ being the tortuosity coefficient of the 3D fluid flow in the corrugated passage. Introducing Eq. (9) in Eq. (8) and taking into account that $\tau=L_{\mathrm{av}} / L$ it follows that:

$$
\frac{\Delta P}{L}=\frac{2 K_{0} \tau^{2} \eta}{D_{\mathrm{H}}^{2}} .
$$

From Eqs. (3), (4), (7) and (10) a mathematical expression that relates the coefficient $K$ from the $f R e$ relation with the shape factor and tortuosity can be obtained:

$K=K_{0} \tau^{2}$.

In the granular beds area, the product $K_{0} \tau^{2}$ is well known as Kozeny's coefficient [26]. Making use of an analogy developed for the laminar flow through granular beds [26,27], the company CIAT developed a model for the design and optimisation of PHEs geometries, $L_{\mathrm{av}}$ being one of the parameters that need to be determined in order to estimate heat transfer coefficients and friction factors [28].

Taking into account the tortuous character of crosscorrugated chevron-type PHEs passages Wanniarachchi et al. [29] proposed, starting from a coefficient $K=24$ for infinite flat parallel plates, a coefficient $K=26.4 / \sin (\beta)$ for fully developed laminar flow in cross-corrugated chevron-type PHEs. Since the flow passage reduces to a collection of tubes for $\beta=90^{\circ}$, the referred authors abandoned the limit of 26.4 and developed an alternate correlation for fully developed laminar flow [29]:

$K=\frac{1774}{\beta^{1.026}}$.

Using this expression for laminar flow and another one for turbulent flow, Wanniarachchi et al. [29] proposed a unified correlation, satisfying the three flow regimes. It is important to note that this pressure loss correlation apply to the loss in the passages alone and exclude any losses in the PHEs entry and exit port manifolds. From the unified correlation is possible to conclude that the critical Reynolds numbers are located in the approximate range 20-100 and that this Reynolds numbers increase as the chevron angle increases, as observed also by Kumar [20].
Palm and Claesson [30] concluded in a literature survey that the existent correlations for single-phase heat transfer and pressure drop in PHEs do not take all geometrical parameters into account and that there is still a need for the verification of the correlations for values of geometrical parameters differing from those most commonly used. The same authors suggested that numerical calculations using unit cells [31,32] could allow the integration and averaging across the referred cells, resulting in correlations including the influence of all PHEs parameters [30].

In the present work it will be study numerically the coefficient $K$ from the $f R e$ correlations for fully developed laminar flow in cross-corrugated chevron-type PHEs passages. Typical values of the geometric parameters $(\beta$ and $\gamma$ ) will be used to design the chevron-type plates as well as values that provide heat exchangers with high area densities. The numerical values of the tortuosity coefficient and the values of $K_{0}$ allow estimating the coefficient $K$ for the different passages (Eq. (11)).

\section{Model details}

Numerical simulations in the laminar regime were performed resorting to a Newtonian fluid with constant physical properties. The numerical calculations were performed in four steps (Fig. 2), using the commercial finite element software package POLYFLOW ${ }^{\circledR}$. Details associated to these different steps are provided below.

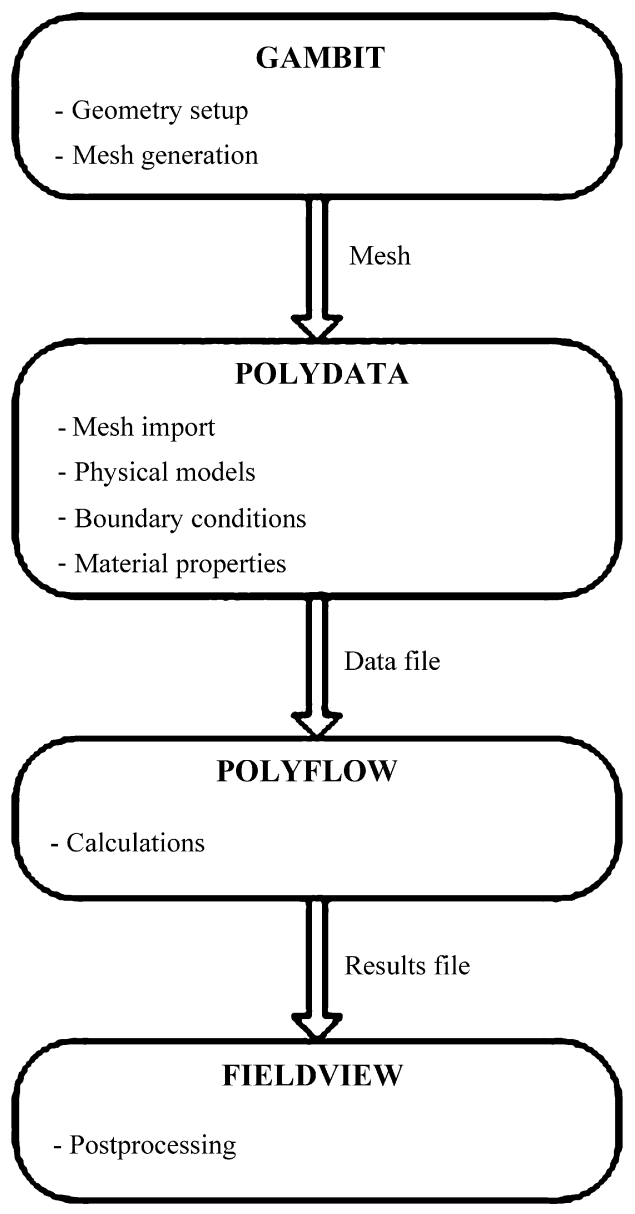

Fig. 2. Program structure 


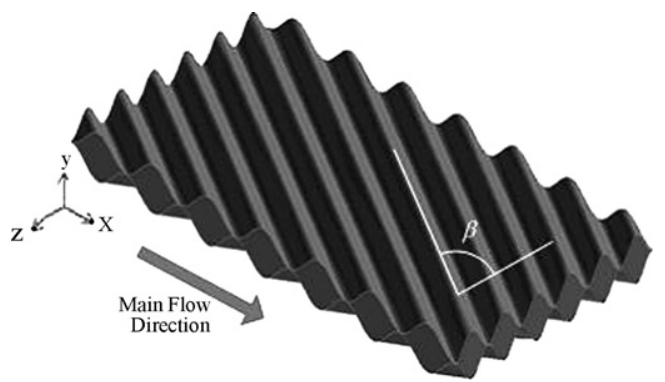

Fig. 3. Representation of half of the PHE channel with plate P6 and $\beta=59^{\circ}$.

\subsection{Geometry setup and mesh generation}

The corrugations were described, in the main flow direction ( $x x$ axis), by a sine curve [19,31]:

$y(x)=\frac{b}{2} \sin \left(\frac{2 \pi}{p_{x}}\left(x-\frac{p_{x}}{4}\right)\right)+\frac{b}{2}$.

In the present work the corrugation angle for the distinct plates assumed the values: $29.0^{\circ}, 39.8^{\circ}, 48.0^{\circ}, 59.0^{\circ}, 74.5^{\circ}$ and $84.9^{\circ}$. For each corrugation angle, the geometrical properties varied as summarized in Table 1.

In previous studies [19,33-35], a uniform flow was considered inside each channel and, for this reason, a symmetry axis was established (Fig. 1) simplifying the geometrical domain to half of a channel (Fig. 3). This type of geometry was used in the referred investigations on the numerical simulation of the laminar non-isothermal flow of yoghurt [33], and their numerical results were in very good agreement with the experimental data from Afonso et al. [7].

Analysing the results obtained in the referred studies, it was possible to observe the periodicity of the flow along the width ( $z z$ axis) of the channel, which allowed the simplification of the geometrical domain to the channel represented in Fig. 4.

The geometrical periodicity in the width of the channel could also be observed on its length. Periodic unitary cells (Fig. 5(a)), including 5 contact points between the plates -1 in the middle and 4 in the corners (Fig. 5(b)) - could be also found along the length of the channel. The concept of unitary cell has already been used by different authors [31,32]. In these works the unitary cell was bound by 4 adjacent contact points while the one used

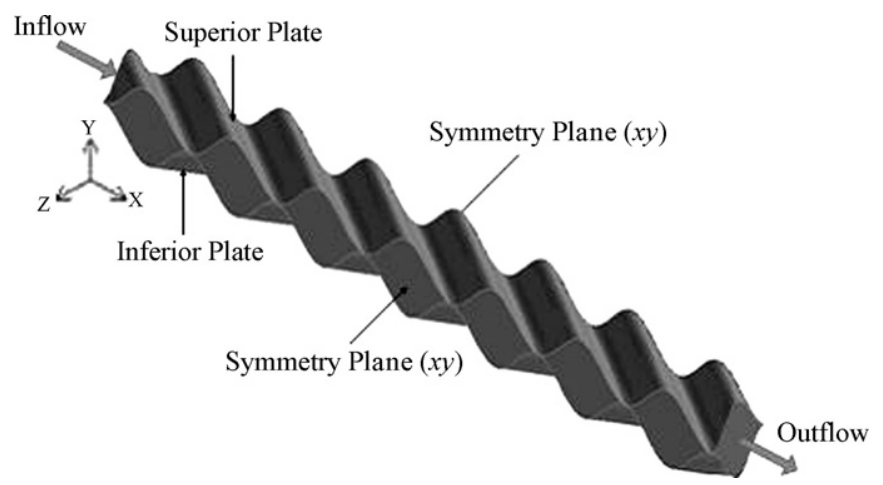

Fig. 4. Representation of the computational domain used in the present work for the PHE channel obtained with plate P6 and $\beta=59^{\circ}$. in the present work includes an additional contact point in the centre.

Local Fanning friction factors were calculated in each of the consecutive unitary cells an asymptotic value (hydrodynamic fully developed flow) being found on the fifth or sixth cell. Since the last cell was non-periodic, the simulations were performed in channels constituted by seven consecutive unitary cells (Fig. 4).

As in past investigations [19,33-35], due to the complexity of the computational domain, an unstructured mesh constituted by tetrahedral, hexahedral and pyramidal elements was used (Fig. 5). The grid generation procedure can be divided on three steps [36]: first, all the faces that could not be meshed with triangular elements were meshed by means of quadrilateral elements; second, if any quadrilateral face elements exist on the volume faces, pyramidal elements were generated to create the transition from the associated hexahedral/quadrilateral elements to the tetrahedral elements that will occupy the remainder of the volume; and finally, the remainder of the volume was meshed with tetrahedral elements.

As presented in Table 1, the 36 geometries studied in the present work have very distinct geometrical properties. Thus, the size of the grid elements differed from geometry to geometry (the nodal distance on the edges of the volume varied between 0.1 and $0.3 \mathrm{~mm})$.
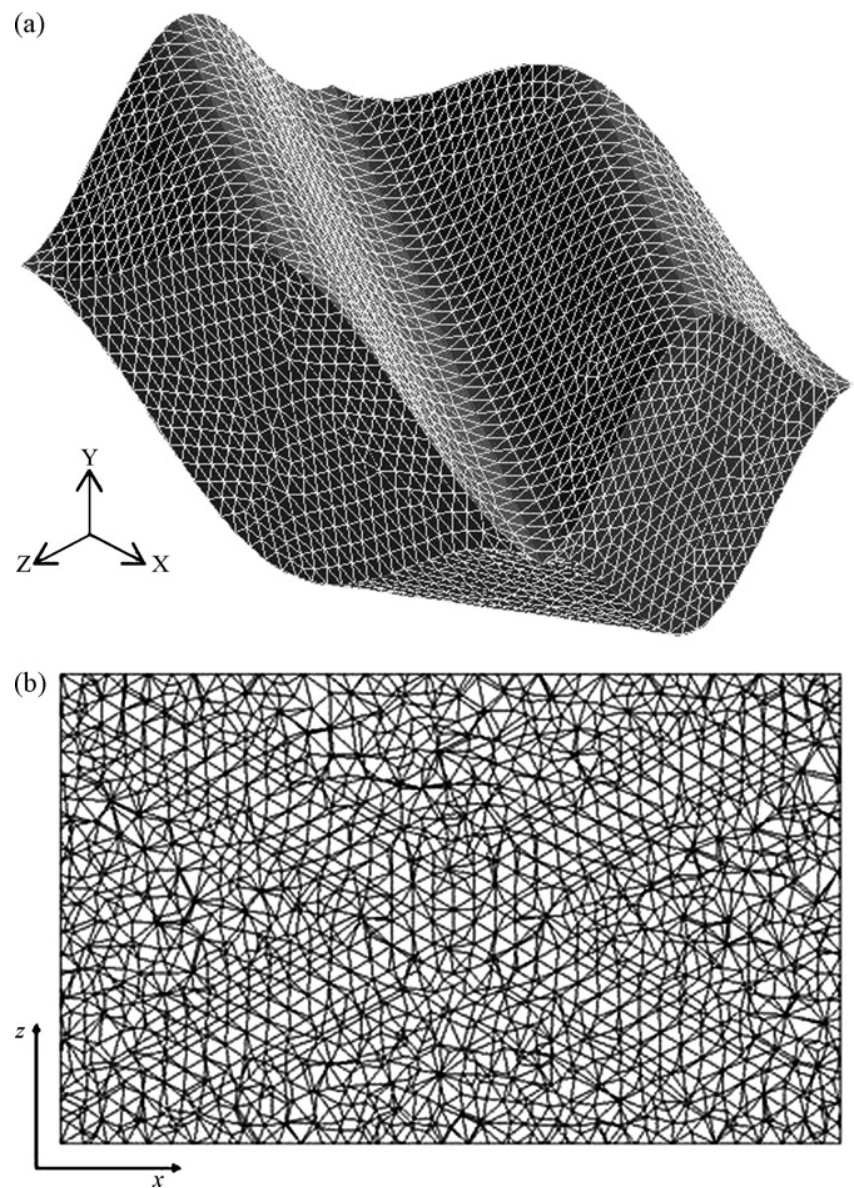

Fig. 5. Unitary cell obtained with plate $\mathrm{P} 3$ and $\beta=59^{\circ}$. (a) Representation of the mesh with nodal distance on the edges $0.3 \mathrm{~mm}$. (b) Mesh in the plane of contact points $(y=0)$ with nodal distance on the edges $0.3 \mathrm{~mm}$. 
Table 1

Geometrical properties of the plates

\begin{tabular}{llll}
\hline Name & $b(\mathrm{~mm})$ & $p_{x}(\mathrm{~mm})$ & $\gamma$ \\
\hline P1 & 1.9 & 10 & 0.38 \\
P2 & 2.6 & 13.7 & 0.38 \\
P3 & 2.6 & 10 & 0.52 \\
P4 & 3.2 & 10 & 0.64 \\
P5 & 2.6 & 6.8 & 0.76 \\
P6 & 3.8 & 10 & 0.76 \\
\hline
\end{tabular}

The size of the elements, for each of the channels, was fixed after a grid independence test. The calculations were carried out using meshes with different element sizes [37-39] and the obtained Fanning friction factors were compared, the results being considered independent of the mesh when a deviation below $1 \%$ [40] was observed.

In Table 2 it is presented the grid independence test for plate $\mathrm{P} 3$ and $\beta=59^{\circ}$ (plate and mesh illustrated in Fig. 5). It can be observed that the value of $K$ obtained using the mesh with 160,910 elements differs less than $1 \%$ of that obtained with the mesh containing 269,380 elements. By this reason the former mesh was chosen to perform the calculations.

For $\beta=60^{\circ}$ Kumar [20] predicts $K=24$, while Wanniarachchi et al. [29] predicts $K=27.0433$ for $\beta=59^{\circ}$ (Eq. (12)). The result presented in Table 2 compares well with these values. In Section 3 it can be observed that the numerical results of $K$ obtained in the region of common used area enlargement factors are in good agreement with literature data for different values of $\beta$.

The unit cell used in the present work includes five contact points between the plates, low velocities being observed in the nearby of these points [41]. The existence of contact points require the use of meshes containing elements small enough (Table 2) in order to be achieved a good agreement between the numerical results and experimental data from the literature.

\subsection{Boundary conditions}

Distinct flow rates were imposed in the plane $x=0$, the outlet being located in the plane $x=l$ (i.e., the total length of the channel).

As described earlier, the geometry used on this work is one of the periodic channels found in the width of one complete channel of a PHE. Since periodicity in the width of the channel was observed, planes of symmetry were imposed on the sides ( $x y$ planes) of the geometry (Fig. 4). In the interfaces between the fluid and the inferior and superior plates (Fig. 4), slip was assumed to be non-existent.

Table 2

Grid independence test

\begin{tabular}{llll}
\hline Nodal distance $(\mathrm{mm})$ & Number of elements & Number of nodes & $K$ \\
\hline 0.25 & 269,380 & 55,691 & 26.9685 \\
0.30 & 160,910 & 34,848 & 27.0013 \\
0.35 & 121,500 & 26,593 & 27.6627 \\
\hline
\end{tabular}

\subsection{Numerical calculations}

The problem was numerically solved using the finite-element computational fluid dynamics software POLYFLOW ${ }^{\circledR}$ and the simulations were performed using a Dell Workstation PWS530 with $1 \mathrm{~GB}$ of RAM.

The equations solved were the conservation of mass and momentum equations for laminar incompressible flow. This problem is a non-linear problem, so it was necessary to use an iterative method to solve the referred equations. In order to evaluate the convergence of this process, a test based on the relative error in velocity field was performed [42], and the convergence test value was set to $10^{-4}[19,33-35]$, i.e., the process is assumed to be convergent on the iteration where $\left\|\mathbf{u}_{i}-\mathbf{u}_{i-1}\right\| /\left\|\mathbf{u}_{i-1}\right\|<10^{-4},\|\mathbf{u}\|=\sqrt{u_{x}^{2}+u_{y}^{2}+u_{z}^{2}}$ being the norm of the velocity vector.

\subsection{Model validation}

In order to verify the reliability and exactness of the model, fully developed laminar flow inside double-sine ducts (Fig. 6) were first studied. The fluid was Newtonian and had constant physical properties. Since Ding and Manglik [1] studied analytically the same problem, the present numerical values of $K$ were compared with the solutions from the referred authors. When the PHE channels have a corrugation angle $\beta=90^{\circ}$, a bundle of straight double-sine ducts are observed.

Three double-sine ducts with distinct ratios $b / a$ (Fig. 7) were used. All of them presented a distance between plates, $b$, of $2.6 \mathrm{~mm}$ and the ratio $b / a$ assumed the values of $0.5,1$ and 2 .

The grid used to perform this study was obtained by the procedure described on Section 2.1 and a representation of the unstructured mesh, on a cross section of a double-sine duct, is shown in Fig. 7. The distribution of the grid elements obtained with these ducts, with $\beta=90^{\circ}$, is similar to the distribution observed in the meshes of the remaining studied geometries (Fig. 5(a)), with corrugation angles located in the interval $29^{\circ}<\beta<85^{\circ}$. In each cross section of the double-sine ducts it can also be observed contact points between the plates.

The simulations were carried out in the conditions detailed in Section 2.2. The numerical results of the coefficient $K$, for fully

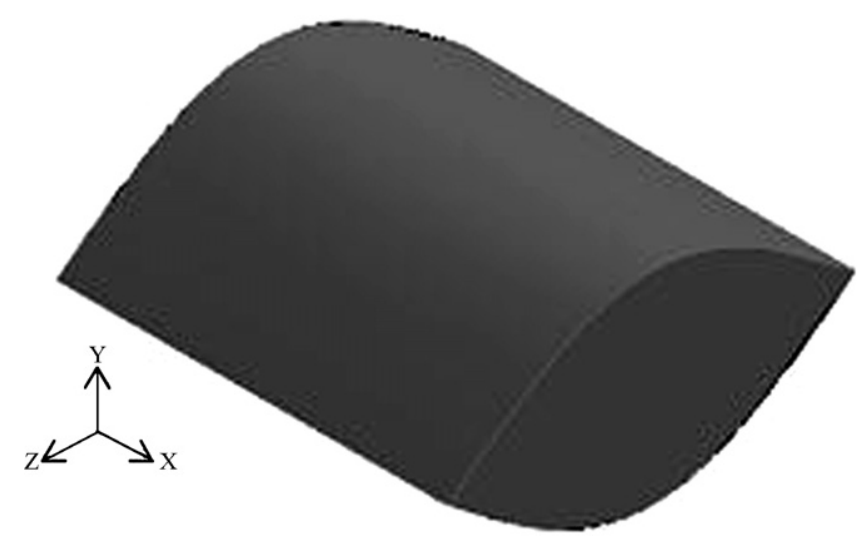

Fig. 6. Double-sine duct with a ratio $(b / a)$ of 0.5 . 


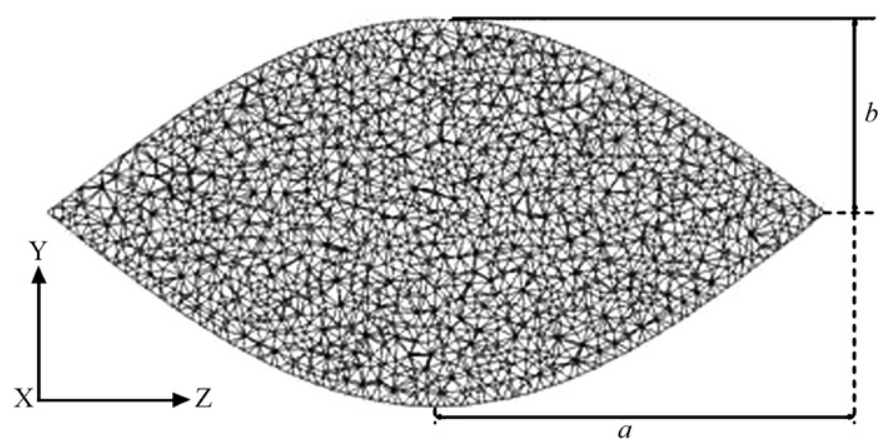

Fig. 7. Double-sine duct with a ratio $(b / a)$ of 0.5 mesh on a cross section.

Table 3

Coefficient $K$ in double-sine ducts

\begin{tabular}{lllll}
\hline b/a & $\begin{array}{l}\text { Nodal distance on the } \\
\text { edges }(\mathrm{mm})\end{array}$ & Computed & Ref. [1] & Difference (\%) \\
\hline 0.5 & 0.22 & 15.0722 & 15.0527 & 0.13 \\
1 & 0.18 & 15.5795 & 15.5744 & 0.03 \\
2 & 0.14 & 16.3196 & 16.2861 & 0.21 \\
\hline
\end{tabular}

developed laminar flow, are summarized on Table 3 and it can be observed that the numerical results compare very well with the analytical solutions from Ding and Manglik [1].

\section{Results}

The heat transfer area can be increased by using larger exchangers, but the more cost-effective way is to use a heat exchanger having a large area density per unit volume [43]. In the present investigation, for $29^{\circ}<\beta<60^{\circ}$ (commonly used chevron angles) and plates $\mathrm{P} 1, \mathrm{P} 2$ and $\mathrm{P} 3$ common area enlargement factors $(1.1<\phi<1.5)$ were obtained. In this range of $\beta$, plates P4, P5 and P6 provided values of higher $\phi$ (up to 1.85). For $\beta>60^{\circ}, \phi$ assumes large values (Fig. 8) for all the studied plates (Table 1).

The area enlargement factors of the studied plates can be estimated by Eq. (2) and geometrical properties from Table 1. These values compare very well with the ones obtained numerically (Fig. 8).

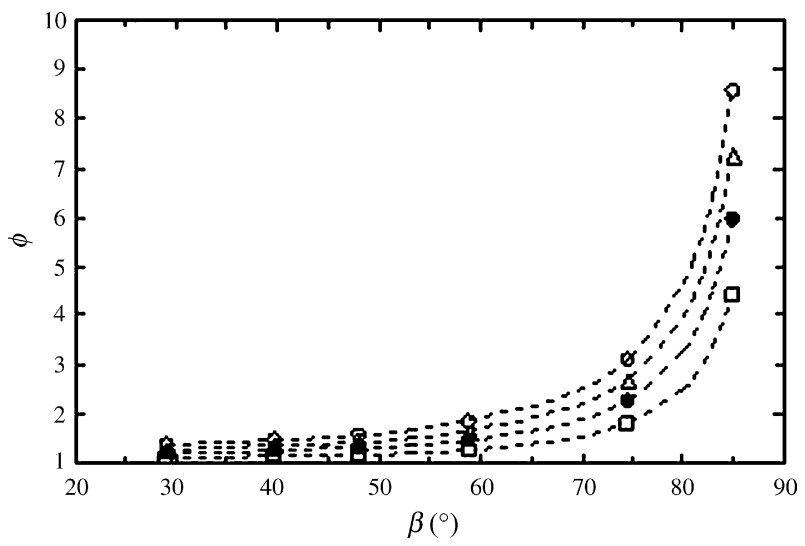

Fig. 8. Area enlargement factor for the different plates. ( $\square$ ) Plate P1; (○) Plate P3; $(\triangle)$ Plate P4 and $(\bigcirc)$ Plate P6. Lines represent Eq. (2).
For a constant chevron angle, $\phi$ increases when the channel aspect ratio in the main flow direction increases (Eq. (2)). The variation of $\gamma$ and $\beta$ in a broad range can be useful to study the behaviour of the tortuosity coefficient, $\tau$. As already mentioned, the company CIAT [28] uses the values of the tortuosity coefficient of the passages in their PHE optimization model. In a first step, horizontal and vertical tortuosity coefficients are measured $\tau$ being obtained by the product of the former coefficients [28].

The horizontal ( $x z$ plane) tortuosity is related with the chevron angle of the PHEs passages. As can be seen in Fig. 9, when the chevron angle is low (Fig. 9(a)) the fluid has a fluid velocity component in the $z$ direction superior to that when the chevron angle is high (Fig. 9(b)). It seems reasonably to admit that the horizontal tortuosity is directly proportional to $1 / \sin (\beta)$, as suggested by Wanniarachchi et al. [29], and easily induced by geometric considerations.

The vertical tortuosity is related with the waving behaviour of the flow on the $x y$ plane provoked by the corrugations, this behaviour being associated to the $\gamma$ values (Eq. (1)). When the value of $\gamma$ is low (Fig. 10(a)) the fluid has a component of the velocity on the $y$ direction inferior to that when the value of $\gamma$
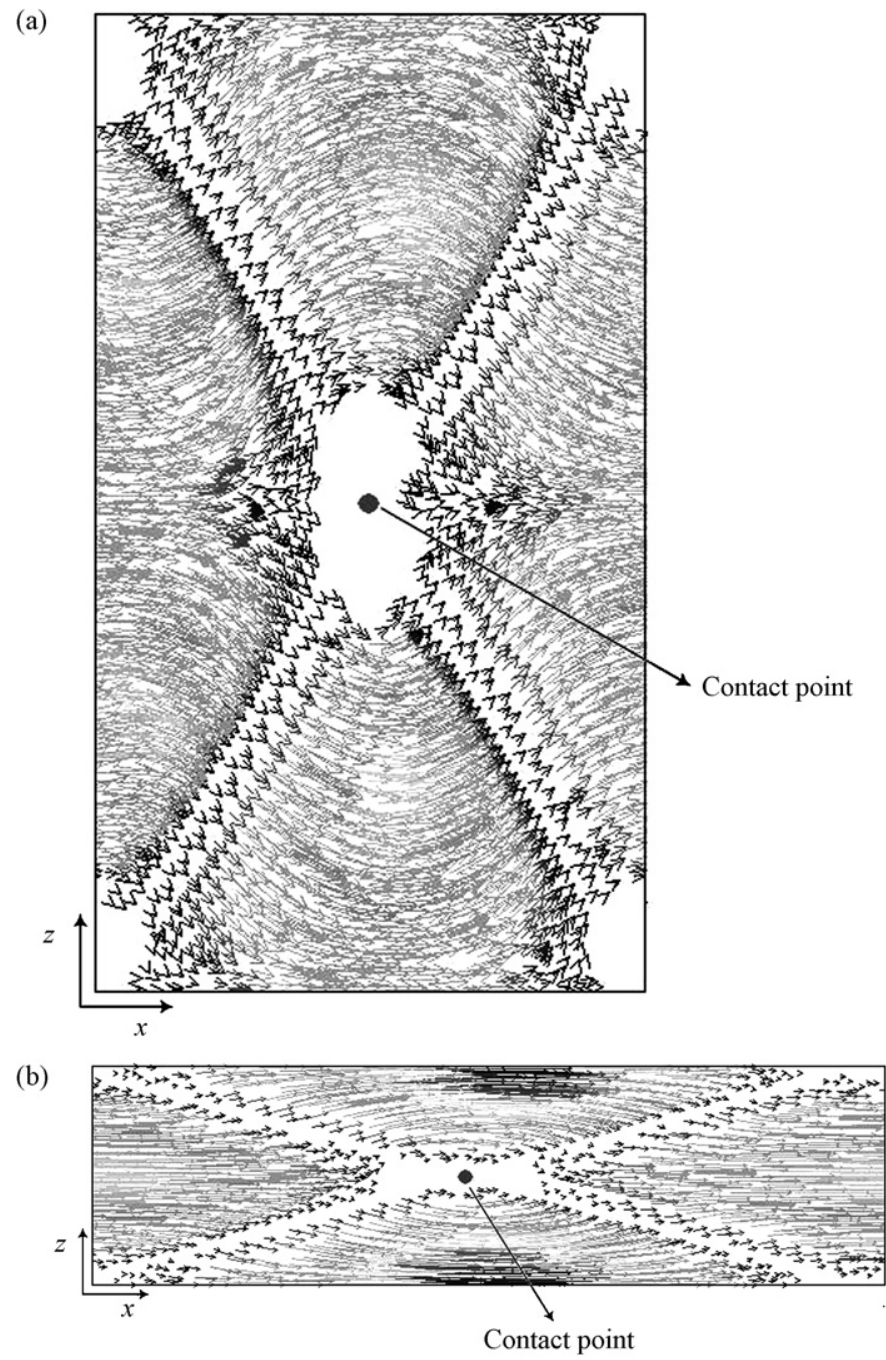

Fig. 9. Velocity vectors, in the plane of the contact points, for PHE channels with plates P5 and different $\beta$. (a) $\beta=29.0^{\circ}$ and (b) $\beta=74.5^{\circ}$. 

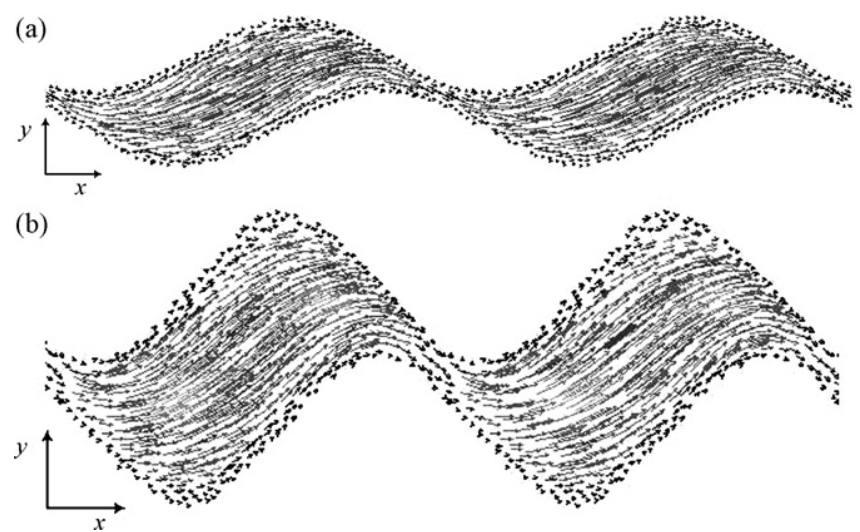

Fig. 10. Velocity vectors in a plane $z=$ cte. for PHE channels with $\beta=29.0^{\circ}$ and different $\gamma$. (a) $\gamma=0.38$ (plate P2) and (b) $\gamma=0.76$ (plate P5).

is high (Fig. 10(b)). Thus, the vertical tortuosity increases with the increase of $\gamma$.

Using POLYFLOW ${ }^{\circledR}$ it was possible to evaluate the average interstitial velocity in fully developed laminar regime for the different passages of Table 1 . Since the mean velocity is known (Eq. (5)), the tortuosity coefficient was calculated by Eq. (9) (Fig. 11). In Fig. 11 it can be observed that the tortuosity coefficient increases with the increase of $\gamma$ and decrease of $\beta$.

As can be seen in Table 1, plates P1 and P2 are characterized by different values of $b$ and $p_{x}$ but produce the same tortuosity coefficients (Fig. 11). This is explained by the fact that these plates presents the same value of $\gamma(0.38)$ and the same can be concluded with respect to plates P5 and P6 $(\gamma=0.76)$. Thus, the geometric parameters $\gamma$ and $\beta$ unambiguously define the tortuosity coefficient.

The tortuosity coefficient was modelled by Eq. (14), resorting to the function $1 / \sin (\beta)$ (related with the horizontal tortuosity) and $\gamma$ (related with the vertical tortuosity).

$\tau=1+0.5 \sqrt{\left(\frac{1}{\sin (\beta)}\right)^{\gamma}-1}$.

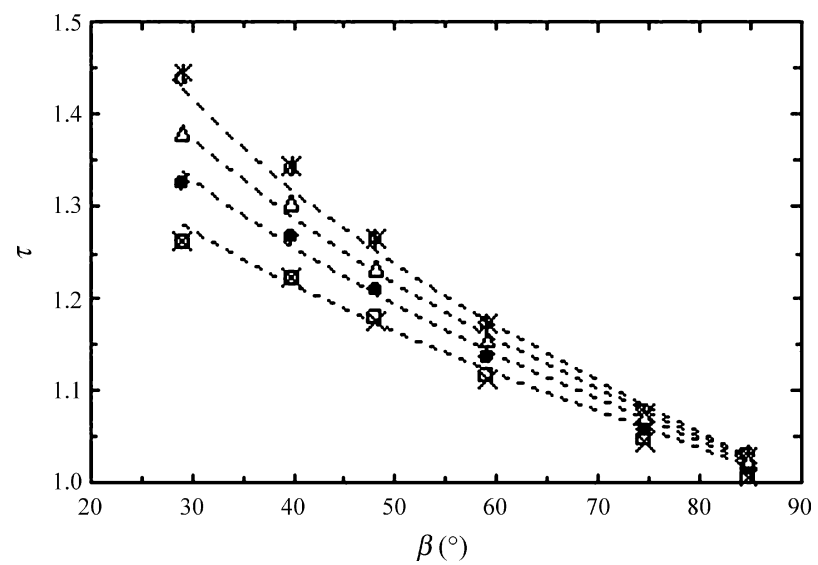

Fig. 11. Tortuosity coefficient for different channels. $(\square)$ Plate P1 $(\gamma=0.38)$; $(\times)$ plate $\mathrm{P} 2(\gamma=0.38) ;(\circlearrowleft)$ plate $\mathrm{P} 3(\gamma=0.52) ;(\triangle)$ plate $\mathrm{P} 4(\gamma=0.64) ;(*)$ plate P5 $(\gamma=0.76)$ and $(\bigcirc)$ plate P6 $(\gamma=0.76)$. Lines represent Eq. (14).
When $\beta$ tends to $90^{\circ}$ the PHEs passages become closer to a collection of straight tubes [1,23,29] and the tortuosity should become closer to one. The numerical results and Eq. (14) express this trend (Fig. 11). The referred equation compares very well with the numerical values (maximum difference of $1.7 \%$ ) and can be used to estimate the tortuosity coefficient of doublesine chevron-type PHEs ducts in the ranges $29^{\circ}<\beta<85^{\circ}$ and $0.38<\gamma<0.76$.

After determining the numerical values of $\tau$, the numerical values of the shape factor (Fig. 12) of each designed duct were calculated by Eq. (10), resorting to the numerical values of $\Delta P$ across a unitary cell, in fully developed laminar regime (in the fifth or sixth consecutive unit cell, Figs. 4 and 5).

As can be seen in Fig. 12, the shape factor is only weakly influenced by $\gamma$, the variations of $K_{0}$ with $\gamma$ being more obvious for the lower angle. The shape factor increases with the decrease of $\beta$ and assumes a value, for $\beta=29^{\circ}$, of about twice that from a cylindrical channel (16). The shape factor can be predicted in the ranges $29^{\circ}<\beta<85^{\circ}$ and $0.38<\gamma<0.76$ - with accuracy (maximum deviation of $3 \%$ ) by the following expression:

$K_{0}=16\left(\frac{90}{\beta}\right)^{0.6554-0.0929 \gamma}$.

In Eq. (15) a reference value of 16 for $K_{0}$ was considered for $\beta=90^{\circ}$, since in all the simulations (six) for $\beta=84.9^{\circ}$ the factor $K_{0}$ was in the range 16-17. However, it is well known [1] that for $\beta=90^{\circ}$ a bundle of double-sine ducts are obtained and the value of $K_{0}$ can fall below 16. This can be seen in Table 3 for the ratios $b / a$ of 0.5 and 1 .

For the sake of clarity of Fig. 12, the curves obtained with Eq. (15) are not included. An approximate value to replace the function $0.6554-0.0929 \gamma$ is 0.6 since this function varies in the range $0.58-0.62$.

Resorting to the numerical values of $\tau$ and $K_{0}$ the numerical values of the coefficient $K$ were estimated trough Eq. (11) and are shown in Fig. 13. In this figure the curves obtained by Eq. (11), with $\tau$ given by Eq. (14) and $K_{0}$ given by Eq. (15) are also shown. The numerical values of $K$ are well described by the analytical model (maximum difference of $3.5 \%$ ).

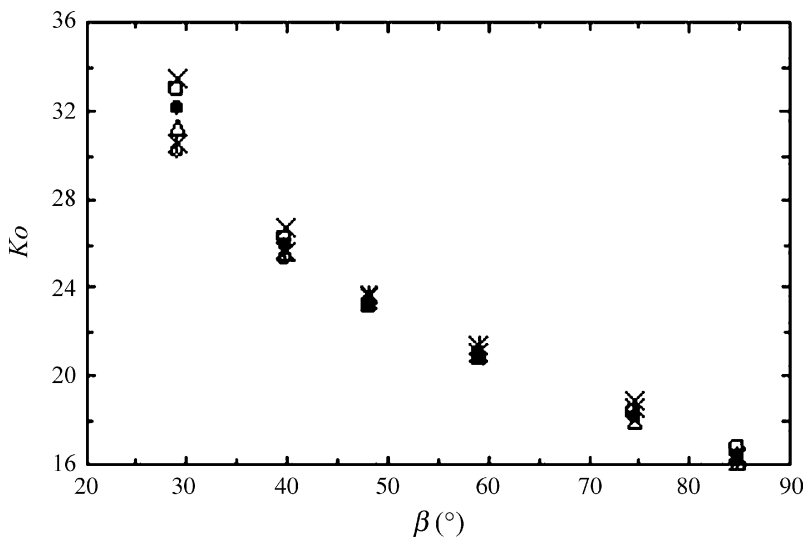

Fig. 12. Shape factor for the different channels. ( $\square)$ PLATE P1; $(\times)$ plate P2; $(\bullet)$ plate $\mathrm{P} 3 ;(\triangle)$ plate $\mathrm{P} 4,(*)$ plate $\mathrm{P} 5$ and $(\bigcirc)$ plate $\mathrm{P} 6$. 


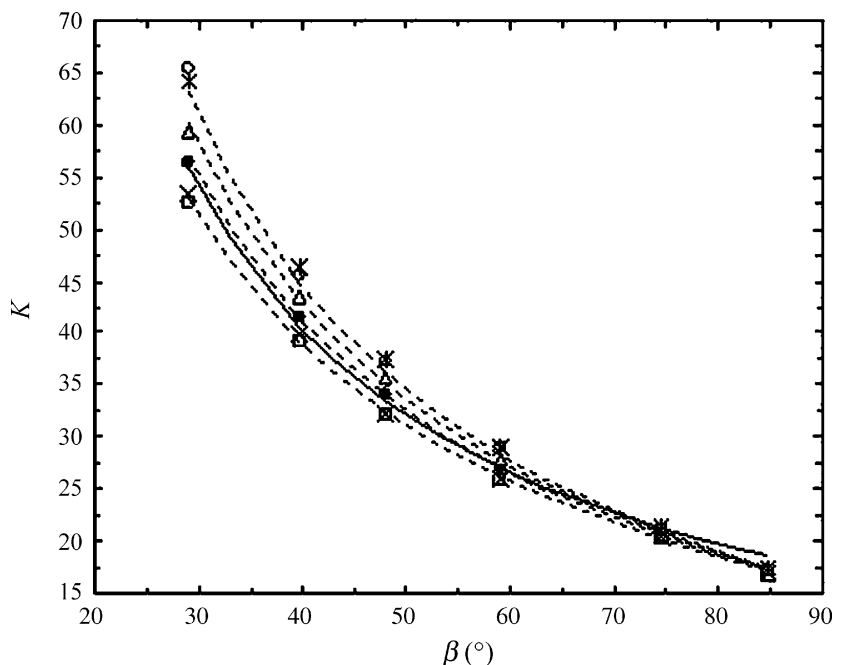

Fig. 13. Coefficient $K$ for different channels. $(\square)$ Plate $\mathrm{P} 1 ;(\times)$ plate $\mathrm{P} 2$; plate P3; $(\triangle)$ plate $\mathrm{P} 4,(*)$ plate $\mathrm{P} 5$ and $(\bigcirc)$ plate P6. Lines (-- ) represent Eq. (11) with $\tau$ given by Eq. (14) and $K_{0}$ given by Eq. (15). Line (-) represents Eq. (12).

The coefficient $K$ is also unambiguously defined by $\gamma$ and $\beta$, as it was found for the shape factor and tortuosity coefficient. For $\beta=75^{\circ}$ and $84.5^{\circ}$, plates with very different geometrical properties (Table 1 and Fig. 8) give rise to similar coefficients $K$. This coefficient is much more sensitive to PHEs geometrical properties variations for low corrugation angles.

In the $K$ coefficient case it is possible to try to compare the present results with literature data, although this is scarce in the laminar region. For example, Ayub [22] recommended the relations from Wanniarachchi et al. [29] for elaborate calculations, namely Eq. (12).

In Fig. 13 it is possible to observe that Eq. (12) is located within the bounds found in the present work for $\gamma=0.38$ and 0.52 when the corrugation angle is located in commonly used $\beta$ range $30-60^{\circ}$. Since in this region, $\phi$ varies (in the present investigation) between 1.1 and 1.47 (the usual range) and Eq. (12) was developed for this $\phi$ region, there is a good agreement between the present calculations and Eq. (12). For $\beta>70^{\circ}$ (a not commonly studied region) the values of $K$ found in the present investigation are lower than the predicted by Wanniarachchi et al. [29].

\section{Concluding remarks}

Numerical simulations and unitary cells with five contact points between plates were used to predict the hydraulic performance trends, in fully developed laminar flow, with varying the geometrical properties of PHEs passages.

With the help of geometrical considerations, the numerical values of the tortuosity coefficient were modelled. Resorting to the tortuosity coefficients and shape factor of the ducts the coefficient from the fRe correlations was also modelled. The models proposed for these coefficients take into account their dependence on the corrugation angle and channel aspect ratio.

The channel aspect ratio and corrugation angle unambiguously define the tortuosity coefficient, shape factor and the coefficient from $f R e$ correlations. The tortuosity coefficient and the coefficient $K$ from the friction factor correlations increase with the increase of the channels aspect ratio and the decrease of the chevron angle. The shape factor from the PHEs passages increase with the decrease of the chevron angle and is weakly influenced by the channel aspect ratio.

The present study can be useful in order to determine pressure drops in PHEs channels, convective heat transfer coefficients, prediction of port-to-channel flow maldistribution, estimation of wall shear rates and definition of generalised Reynolds numbers that allow the establishment of a single friction curve equation for both Newtonian and power law fluids, in different flow regimes.

\section{Appendix A. Nomenclature}

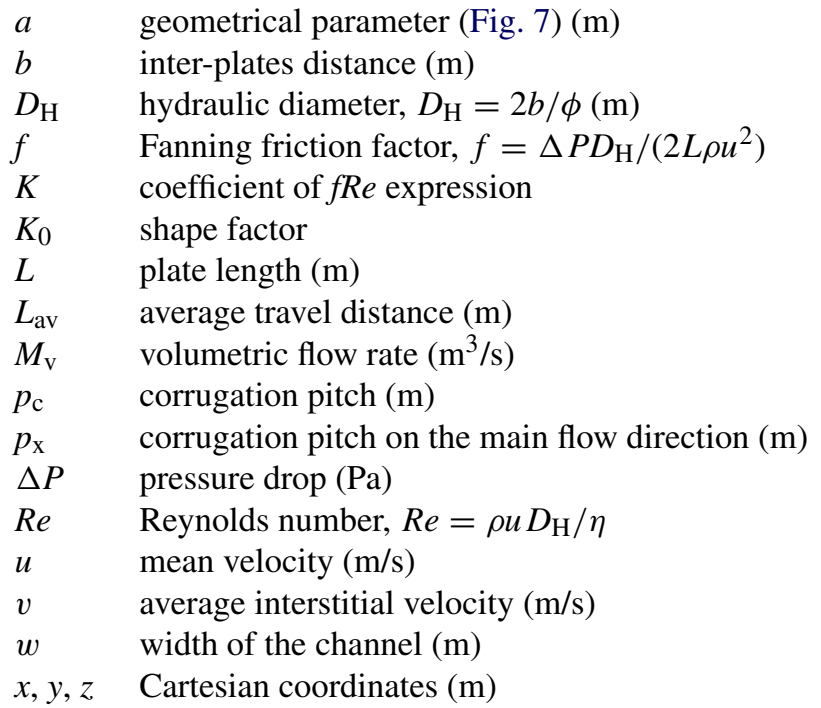

\section{Greek letters}

$\beta \quad$ corrugation angle $\left({ }^{\circ}\right)$

$\phi \quad$ area enlargement factor (Eq. (2))

$\gamma \quad$ channel aspect ratio on the main flow direction, $\gamma=$ $2 b / p_{x}$

$\eta \quad$ fluid viscosity (Pa s)

$\rho \quad$ fluid density $\left(\mathrm{kg} / \mathrm{m}^{3}\right)$

$\tau \quad$ tortuosity

\section{References}

[1] J. Ding, R.M. Manglik, Analytical solutions for laminar fully developed flows in double-sine shaped ducts, Heat Mass Trans. 31 (1996) 269-277.

[2] L. Fischer, H. Martin, Friction factors for fully developed laminar flow in ducts confined by corrugated parallel walls, Int. J. Heat Mass Transfer 40 (1996) 635-639.

[3] B. Farhanieh, B. Sunden, Three-dimensional laminar flow and heat transfer in the entrance region of trapezoidal ducts, Int. J. Numer. Methods Fluids 13 (1991) 537-556.

[4] J.Y. Murthy, S. Mathur, Periodic flow and heat transfer using unstructured meshes, Int. J. Numer. Methods Fluids 25 (1997) 659-677.

[5] R.K. Shah, Laminar flow friction and forced convection heat transfer in ducts of arbitrary geometry, Int. J. Heat Mass Transfer 18 (1975) 849-862. 
[6] P.S. Lee, S.V. Garimella, Thermally developing flow and heat transfer in rectangular microchannels of different aspect ratios, Int. J. Heat Mass Transf. 49 (2006) 3060-3067.

[7] I.M. Afonso, L. Hes, J.M. Maia, L.F. Melo, Heat transfer and rheology of stirred yoghurt during cooling in plate heat exchangers, J. Food Eng. 57 (2003) 179-187.

[8] F.C.C. Galeazzo, R.Y. Miura, J.A.W. Gut, C.C. Tadini, Experimental and numerical heat transfer in plate heat exchanger, Chem. Eng. Sci. 61 (2006) 7133-7138.

[9] P. Gschwind, G. Gaiser, C. Zimmerer, V. Kottke, Transport phenomena in micro heat exchangers with corrugated walls, Microscale Therm. Eng. 5 (2001) 285-292.

[10] H.M. Metwally, R.M. Manglik, Computational modelling of enhanced laminar flow heat transfer in viscoplastic fluids in corrugated-plate channels, in: Proceedings ASME International Mechanical Engineering Congress \& Exposition, New Orleans, Louisiana, 2002, pp. 1-8.

[11] J.C. Leuliet, J.F. Maingonnat, M. Lalande, Thermal behaviour of plate heat exchangers with Newtonian and non-Newtonian fluids, in: Proceedings Congrés Eurotherm 5 et 1er Colloque TIFAN, Compiègne, 1988, pp. 1-15.

[12] J.C. Leuliet, J.F. Maingonnat, M. Lalande, Etude de la perte de charge dans des échangeurs de chaleur à plaques traitant des produits non-newtoniens, Rev. Gén. Therm. 308-309 (1987) 445-450.

[13] M.K. Bassiouny, H. Martin, Flow distribution and pressure drop in plate heat exchangers-I, Chem. Eng. Sci. 39 (1984) 693-700.

[14] B.P. Rao, P.K. Kumar, S.K. Das, Effect of flow distribution to the channels on the thermal performance of a plate heat exchanger, Chem. Eng. Process. 41 (2002) 49-58.

[15] F. Delplace, J.C. Leuliet, Generalized Reynolds Number for the flow of power law fluids in cylindrical ducts of arbitrary cross-section, Chem. Eng. J. 56 (1995) 33-37.

[16] A.C.A. Gratão, V. Silveira Jr., J. Telis-Romero, Laminar flow of soursop juice through concentric annuli: friction factors and rheology, J. Food Eng. 78 (2007) 1343-1354.

[17] Y.S. Fangary, M. Barigou, J.P.K. Seville, Simulation of yoghurt flow and prediction of its end-of-process properties using rheological measurements, Trans. Inst. Chem. Eng. 77 (1999), pp. Part C. 33-39.

[18] P. Perona, R. Conti, S. Sordo, Influence of turbulent motion on structural degradation of fruit purees, J. Food Eng. 52 (2002) 397-403.

[19] C.S. Fernandes, R. Dias, J.M. Nóbrega, I.M. Afonso, L.F. Melo, J.M. Maia, Simulation of stirred yoghurt processing in plate heat exchangers, J. Food Eng. 69 (2005) 281-290.

[20] H. Kumar, The plate heat exchanger: construction and design, in: Proceedings First UK National Conference on Heat Transfer, University of Leeds, Inst. Chem. Symp. Series No. 86, 1984, pp. 1275-1288.

[21] S. Kakaç, H. Liu, Heat Exchangers Selection, Rating, and Thermal Design, second ed., CRC Press, Boca Raton, 2002, pp. 373-412.

[22] Z.H. Ayub, Plate heat exchanger survey and new heat transfer and pressure drop correlations for refrigerant evaporators, Heat Transfer Eng. 24 (2003) $3-16$.

[23] H. Martin, A theoretical approach to predict the performance of chevrontype plate heat exchangers, Chem. Eng. Process. 35 (1996) 301-310.

[24] M. Reppich, Use of high performance plate heat exchangers in chemical and process industries, Int. J. Thermal Sci. 38 (1999) 999-1008.

[25] J. Du, X. Hu, W. Wu, B.X. Wang, A thermal dispersion model for single phase flow in porous media, Heat Transfer Asian Res. 32 (2003) 545-551.
[26] R. Dias, J.A. Teixeira, M. Mota, A. Yelshin, Tortuosity variation in a low density binary particulate bed, Sep. Purif. Technol. 51 (2006) 180184.

[27] R.P. Dias, C.S. Fernandes, M. Mota, J.A. Teixeira, A. Yelshin, Permeability and effective thermal conductivity of bisized porous media, Int. J. Heat Mass Transfer 50 (2007) 1295-1301.

[28] O. Charre, R. Jurkowski, A. Bailly, S. Meziani, M. Altazin, General model for plate heat exchanger performance prediction, J. Enhanced Heat Transfer 9 (2002) 181-186.

[29] A.S. Wanniarachchi, U. Ratnam, B.E. Tilton, K. Dutta-Roy, Approximate correlations for chevron-type plate heat exchangers, in: Proceedings ASME HTD, vol. 314, 1995 National Heat Transfer Conference, vol. 12, 1995, pp. $145-151$.

[30] B. Palm, J. Claesson, Plate heat exchangers: calculation methods for singleand two-phase flow, Heat Transfer Eng. 27 (2006) 88-98.

[31] M.A. Mehrabian, R. Poulter, Hydrodynamics and thermal characteristics of corrugated channels: computational approach, Appl. Math. Modell. 24 (2000) 343-364.

[32] M. Ciofalo, J. Stasiek, M.W. Collins, Investigation of flow and heat transfer in corrugated passages-II. Numerical simulation, Int. J. Heat Mass Transfer 39 (1996) 165-192.

[33] C.S. Fernandes, R.P. Dias, J.M. Nóbrega, I.M. Afonso, L.F. Melo, J.M. Maia, Thermal behaviour of stirred yoghurt during cooling in plate heat exchangers, J. Food Eng. 76 (2006) 433-439.

[34] C.S. Fernandes, R.P. Dias, J.M. Nóbrega, J.M. Maia, Effect of corrugation angle on the hydrodynamic behaviour of power-law fluids during a flow in plate heat exchangers, in: Proceedings Fifth International Conference on Enhanced, Compact and Ultra-Compact Heat Exchangers: Science, Engineering and Technology, Whistler, Canada, 2005, pp. 491495.

[35] C.S. Fernandes, R.P. Dias, J.M. Nóbrega, J.M. Maia, Effect of corrugation angle on the thermal behaviour of power-law fluids during a flow in plate heat exchangers, in: Proceedings Fifth International Conference on Enhanced, Compact and Ultra-Compact Heat Exchangers: Science, Engineering and Technology, Whistler, Canada, 2005, pp. 496-501.

[36] GAMBIT 2 Modelling Guide, vol. 1.2, Fluent Inc., Lebanon, USA, 2001, pp. $160-162$.

[37] A. Thakker, T.S. Dhanasekaran, Experimental, computational analysis on guide vane losses of impulse turbine for wave energy conversion, Renew. Energy 30 (2005) 1359-1372.

[38] T.W.H. Sheu, H.P. Rani, Exploration of vortex dynamics for transitional flows in three-dimensional backward-facing step channel, J. Fluid Mech. 550 (2006) 61-83.

[39] A. Valencia, A. Zarate, M. Galvez, L. Badilla, Non-Newtonian blood flow dynamics in a right internal carotid artery with a secular aneurysm, Int. J. Numer. Methods Fluids 50 (2006) 751-764.

[40] H.M. Metwally, R.M. Manglik, Enhanced heat transfer due to curvatureinduced lateral vortices in laminar flows in sinusoidal corrugated-plate channels, Int. J. Heat Mass Transfer 47 (2004) 2283-2292.

[41] T.K. Kho, H. Müller-Steinhagen, An experimental and numerical investigation of heat transfer fouling and fluid flow in flat plate heat exchangers, Trans. Inst. Chem. Eng. 77 (1999) 124-130.

[42] POLYFLOW 3.9 User's Guide, Fluent Inc., Lebanon, USA, 2001, p. 3.

[43] P. Stehlík, V.V. Wadekar, Different strategies to improve industrial heat exchange, Heat Transfer Eng. 23 (2002) 36-48. 